\title{
Weighted Castelnuovo-Mumford Regularity and Weighted Global Generation
}

\author{
F. Malaspina and G.K. Sankaran \\ Politecnico di Torino \\ Corso Duca degli Abruzzi 24, 10129 Torino, Italy \\ e-mail: francesco.malaspina@polito.it \\ University of Bath \\ Bath BA2 7AY, England \\ e-mail: gks@maths.bath.ac.uk
}

September 24, 2018

\begin{abstract}
We introduce and study a notion of Castelnuovo-Mumford regularity suitable for weighted projective spaces.
\end{abstract}

\section{Introduction}

In chapter 14 of [13] Mumford introduced the concept of regularity for a coherent sheaf on a projective space $\mathbb{P}^{n}$. It was soon clear that it was a key notion and a fundamental tool in many areas of algebraic geometry and commutative algebra.

From the algebraic geometry point of view, regularity measures the complexity of a sheaf: the regularity of a coherent sheaf is an integer that estimates the smallest twist for which the sheaf is generated by its global sections. In Castelnuovo's much earlier version, if $X$ is a closed subvariety of projective space and $H$ is a general hyperplane, one uses linear systems (seen now as a precursor of sheaf cohomology) to get information about $X$ from information about the intersection of $X$ with $H$ plus other geometrical or numerical assumptions on $X$.

From the computational and commutative algebra point of view, the regularity is one of the most important invariants of a finitely generated

Mathematics Subject Classification 2010: 14F05, 14J60.

Keywords: Castelnuovo-Mumford regularity, weighted projective spaces. 
graded module over a polynomial ring. Roughly, it measures the amount of computational resources that working with that module requires. More precisely the regularity of a module bounds the largest degree of the minimal generators and the degree of syzygies.

Extensions of this notion have been proposed over the years to handle other ambient varieties instead of projective space: Grassmannians [1, quadrics [2, multiprojective spaces [3, 7], $n$-dimensional smooth projective varieties with an $n$-block collection [7], and abelian varieties [14].

In all these cases the ambient variety is smooth. Maclagan and Smith [12] gave a variant of multigraded Castelnuovo-Mumford regularity, motivated by toric geometry, which applies to some singular varieties: for a different approach to multigraded regularity, see [15].

Since it often happens that a variety can be conveniently embedded in a weighted projective space but embedding it in projective space requires some arbitrary choices, or the use of many variables or high degree equations, it is worthwhile to be able to import these ideas into weighted projective spaces.

The first aim of this project is to introduce and study a notion of regularity, and a related notion of globally generated sheaf, using Koszul complexes, for weighted projective spaces. The theory of [12] applies to weighted projective spaces, but as theirs is a general theory for all toric varieties, we believe that it should be possible to do better in this narrower context. In particular we want the structure sheaf to be regular, which in general does not happen in [12. Specifically, the definitions in [12, applied to weighted projective spaces, take no account of the individual weights, and the results are therefore only those that hold for all weighted projective spaces (and more), irrespective of the weights.

\section{Generalities}

Fix a weighted projective space $\mathbb{P}=\mathbb{P}\left(w_{0}, \ldots, w_{n}\right)$, which we always write with the weights in decreasing order, $w_{0} \geq \cdots \geq w_{n}$. There is a natural quotient map $\pi: \mathbb{P}^{n} \rightarrow \mathbb{P}$ (see [5, Thm. 3A.1]).

We want to follow [6] and regard $\mathbb{P}$ as a graded scheme: see Remark 1.1 . An alternative would be to regard $\mathbb{P}$ as a stack, as in [9, Example 7.27]. If we were interested only in schemes (or varieties), we could assume that the weights $\left(w_{0}, \ldots, w_{n}\right)$ are reduced, i.e. no prime divides $n$ of them, because every weighted projective space is isomorphic as a scheme to a weighted projective space with reduced weights (see [5, Prop. 3C.5]). If we were interested in orbifolds we could similarly assume that $\operatorname{hcf}\left(w_{0}, \ldots, w_{n}\right)=1$. However, the coordinate hyperplanes $\mathbb{H}_{j}$ (see Lemma 2.9 below) do not inherit these conditions, so we must continue to allow arbitrary weights.

For a subset $I=\left\{\nu_{1}, \ldots, \nu_{s}\right\} \subset\{0, \ldots, n\}$ with $0 \leq \nu_{1}<\cdots<\nu_{s} \leq n$, we set $\left|w_{I}\right|=\sum_{\nu \in I} w_{\nu}$. For convenience, we also write $\mathbf{w}_{i}$ for the sum of 
the $i+1$ largest weights, $\mathbf{w}_{i}=w_{0}+\ldots+w_{i}=\left|w_{0, \ldots, i}\right|$, and we write $\mathbf{w}$ for the total weight, $\mathbf{w}=\mathbf{w}_{n}$.

As a $K$-scheme, $\mathbb{P}\left(w_{0}, \ldots, w_{n}\right)=\operatorname{Proj} K\left[x_{0}, \ldots, x_{n}\right]$ with grading given by $\operatorname{deg} x_{i}=w_{i}$. Accordingly, we can define the twisting sheaf $\mathcal{O}_{\mathbb{P}}(1)$ and twists $\mathcal{E}(t)$ for any coherent sheaf $\mathcal{E}$ and $t \in \mathbb{Z}$ : see [5]. In particular we have $\omega_{\mathbb{P}}=\mathcal{O}_{\mathbb{P}}(-\mathbf{w})$.

Remark 1.1. We consider $\mathbb{P}$ as a graded scheme, and the coherent sheaves are, from now on, to be understood as graded sheaves. The reason for this choice is that by working with graded sheaves on $\mathbb{P}$ one avoids all the pathologies of $\mathbb{P}$, because the sheaves of the form $\mathcal{O}_{\mathbb{P}}(j)$ are always invertible: see [6. Chapter 2, p. 30].

With this convention, for $\mathcal{E}$ a coherent sheaf on $\mathbb{P}$, we have $\mathcal{E} \otimes \mathcal{O}_{\mathbb{P}}(j) \cong$ $\mathcal{E}(j)$ for any integer $j$, so we define the modules

$$
H^{i}(\mathcal{E}(\geq l))=\bigoplus_{t \geq l} H^{i}(\mathbb{P}, \mathcal{E}(t))
$$

and similarly $H^{i}(\mathcal{E}(\leq l))$, for $l \in \mathbb{Z}$. We also use the notation

$$
H_{*}^{i}(\mathbb{P}, \mathcal{E})=\bigoplus_{t \in \mathbb{Z}} H^{i}(\mathbb{P}, \mathcal{E}(t))=H^{i}(\mathcal{E}(>-\infty)) .
$$

Observe that $\pi_{*} \mathcal{O}_{\mathbb{P}^{n}}$ is a split vector bundle on $\mathbb{P}$ by [ 5 , Cor. 3A.2]. More precisely

$$
\pi_{*} \mathcal{O}_{\mathbb{P}^{n}} \cong \bigoplus_{\left(r_{0}, \ldots, r_{n}\right)} \mathcal{O}_{\mathbb{P}}(-\mathbf{r}) .
$$

where the sum is over all $\left(r_{0}, \ldots, r_{n}\right)$ such that $0 \leq r_{j}<w_{j}$ for all $j=$ $0, \ldots, n$, and $\mathbf{r}=\sum_{j=0}^{n} r_{j}$.

Lemma 1.2. For any $i \in \mathbb{N}$, if $\mathcal{E}$ is a vector bundle on $\mathbb{P}$ then $H_{*}^{i}(\mathbb{P}, \mathcal{E})=0$ if and only if $H_{*}^{i}\left(\mathbb{P}^{n}, \pi^{*} \mathcal{E}\right)=0$.

Proof. Since $\pi$ is a finite morphism, we have $H_{*}^{i}\left(\mathbb{P}^{n}, \pi^{*} \mathcal{E}\right) \cong H_{*}^{i}\left(\mathbb{P}, \pi_{*} \pi^{*} \mathcal{E}\right)$, and it is enough to observe that (using the projection formula and (3) )

$$
\pi_{*} \pi^{*} \mathcal{E} \cong \mathcal{E} \otimes \pi_{*} \mathcal{O}_{\mathbb{P}^{n}} \cong \mathcal{E} \otimes\left(\bigoplus_{\left(r_{0}, \ldots, r_{n}\right)} \mathcal{O}_{\mathbb{P}}(-\mathbf{r})\right) \cong \bigoplus_{\left(r_{0}, \ldots, r_{n}\right)} \mathcal{E}(-\mathbf{r}),
$$

with notation as in (3).

\section{Weighted Castelnuovo-Mumford Regularity}

We begin by recalling the usual definition of Castelnuovo-Mumford regularity on projective space: see, for example, [11, Chapter 1.8]. 
Definition 2.1. A coherent sheaf $\mathcal{F}$ on $\mathbb{P}^{n}$ is said to be $m$-regular, for $m \in \mathbb{Z}$, if

$$
H^{i}(\mathcal{F}(m-i))=0
$$

for $i=1, \ldots, n$.

It is well known (see [11, Theorem 1.8.3]) that being $m$-regular implies, in particular, that $H^{0}(\mathcal{F}(m+1)) \neq 0$, and in fact much more than that: it is globally generated (and this even holds for $\mathcal{F}(m)$ ).

Maclagan and Smith in 12 gave a definition of regularity for simplicial toric varieties. We refer to it as toric regularity. On $\mathbb{P}$ it reduces to the following

Definition 2.2. Let $\mathbb{P}=\mathbb{P}\left(w_{0}, \ldots, w_{n}\right)$ and $k=\operatorname{lcm}\left(w_{0}, \ldots, w_{n}\right)$. A coherent sheaf $\mathcal{F}$ on $\mathbb{P}$ is said to be $m$-toric regular if, for $i=1, \ldots, n$

$$
H^{i}(\mathcal{F}(m-i k))=0 .
$$

Here we have taken $\mathcal{C}=\{\mathcal{O}(k)\}$ in [12, Definition 6.2]: according to the definition of $\mathcal{C}[i]$ given in [12, Section 4], $\mathcal{F}$ is toric $m$-regular if $H^{i}(\mathcal{F}(p))=0$ for all $i>0$ and for every $p \in m+(-i \mathcal{O}(k)+\mathcal{C})$. That is, $H^{i}(\mathcal{F}(m-i k+t))=$ 0 for all $i>0$ and for every $t \in k \mathbb{N}$, but it is enough to consider $t=0$.

In our more restricted context, we want a definition that takes account of the individual weights, which toric regularity does not.

Our motivation for the definition we make comes from the Koszul complex. On $\mathbb{P}$ (with, as usual, $w_{0} \geq \cdots \geq w_{n}$ ) we define $\mathcal{A}_{j}=\bigoplus_{|I|=j+1} \mathcal{O}\left(-\left|w_{I}\right|\right)$. Then (see [6, Lemma 2.1.3]) there is a Koszul complex on $\mathbb{P}$ given by

$$
0 \longrightarrow \mathcal{A}_{n} \longrightarrow \mathcal{A}_{n-1} \longrightarrow \ldots \longrightarrow \mathcal{A}_{0} \longrightarrow \mathcal{O} \longrightarrow 0 .
$$

For example, if $\mathbb{P}=\mathbb{P}(5,3,2)$ then the Koszul complex is

$$
\begin{aligned}
& \mathcal{O}(-8) \quad \mathcal{O}(-5) \\
& \oplus \\
& 0 \rightarrow \mathcal{O}(-10) \rightarrow \mathcal{O}(-7) \rightarrow \mathcal{O}(-3) \rightarrow \mathcal{O} \rightarrow 0 . \\
& \oplus \oplus \\
& \mathcal{O}(-5) \quad \mathcal{O}(-2)
\end{aligned}
$$

We give the following definition of weighted Castelnuovo-Mumford regularity.

Definition 2.3. Let $\mathbb{P}=\mathbb{P}\left(w_{0}, \ldots, w_{n}\right)$ with $w_{0} \geq \cdots \geq w_{n}$ and $k=$ $\operatorname{lcm}\left(w_{0}, \ldots, w_{n}\right)$. A coherent sheaf $\mathcal{F}$ on $\mathbb{P}$ is said to be $m$-weighted regular, which we abbreviate to $m$-wregular, if for $i=1, \ldots, n$

$$
H^{i}\left(\mathcal{F}\left(t+(m+1) k-\mathbf{w}_{i}\right)\right)=0
$$


for every $t \geq 0$, and also

$$
H^{0}(\mathcal{F}((m+1) k)) \neq 0 .
$$

We often write wregular to mean 0 -wregular.

We define the wregularity of $\mathcal{F}$, Wreg $(\mathcal{F})$, as the smallest integer $m$ such that $\mathcal{F}$ is $m$-wregular.

Remark 2.4. For $\mathbb{P}=\mathbb{P}^{n}$, wregularity and toric regularity both coincide with the usual notion of Castelnuovo-Mumford regularity.

Indeed, in this case we have $m=0$ and $w_{0}=\cdots=w_{n}=1$, so $k=1$ and $w_{i}=i+1$, so taking $t=0$ in Definition 2.3 we get

$$
H^{i}\left(\mathcal{F}\left(k-\mathbf{w}_{i}\right)\right)=H^{i}(\mathcal{F}(-i)) .
$$

Lemma 2.5. For $\mathbb{P}$ any weighted projective space, $\operatorname{Wreg}\left(\mathcal{O}_{\mathbb{P}}\right)=0$.

Proof. In fact for any $t \in \mathbb{Z}$ we have $H^{i}\left(\mathcal{O}_{\mathbb{P}}(t)\right)=0$ for $0<i<n$, and $H^{0}\left(\mathcal{O}_{\mathbb{P}}(k)\right) \neq 0$. For 0 -wregularity we also need $H^{n}(\mathcal{O}(k-\mathbf{w}))=0$, but this holds because $H^{n}(\mathcal{O}(k-\mathbf{w}))$ is Serre dual to $H^{0}(\mathcal{O}(-k))$, which is zero. (See [5. Section 6B] and [6, Proposition 2.1.4] for Serre duality in this context.)

However, $\mathcal{O}$ is not -1 -wregular because $H^{n}(\mathcal{O}(-\mathbf{w})) \cong H^{0}(\mathcal{O}) \neq 0$.

On the other hand we cannot expect $\mathcal{O}_{\mathbb{P}}$ to be toric regular for arbitrary weights. In fact $H^{n}(\mathcal{O}(-n k)) \cong H^{0}(\mathcal{O}(n k-\mathbf{w}))$ which is non-zero in general. However, $\mathcal{O}(n k)$ is always toric regular.

A significant difference between Definition 2.3 and Definitions 2.1 and 2.2 is that we have imposed a non-vanishing condition, because we lack a counterpart to Mumford's theorem [11, Theorem 1.8.3]: see Example 2.7 below. With this in mind, we make the following definition.

Definition 2.6. A coherent sheaf $\mathcal{F}$ on $\mathbb{P}$ is said to be $m$-semiwregular if for $i=1, \ldots, n$

$$
H^{i}\left(\mathcal{F}\left(t+(m+1) k-\mathbf{w}_{i}\right)\right)=0
$$

for every $t \geq 0$.

Example 2.7. If $\mathbb{P}=\mathbb{P}(3,2)$ then $\mathcal{O}_{\mathbb{P}}(-5)$ is 0 -semiwregular but not 0 wregular, whereas $\mathcal{O}_{\mathbb{P}}(-4)$ is 0 -wregular.

In fact, $m=0$ and $k=6$, so $H^{1}(\mathcal{O}(-5+6-5)) \cong H^{0}(\mathcal{O}(-1))=0$, and thus if we take $\mathcal{F}=\mathcal{O}(-5)$ then the condition $H^{1}(\mathcal{O}(-5) \otimes \mathcal{O}(t+6-(3+$ $2)))=0$ is satisfied for every $t \geq 0$, but $H^{0}(\mathcal{O}(-5) \otimes \mathcal{O}(k))=H^{0}(\mathcal{O}(1))=0$.

On the other hand, for $\mathcal{O}(-4)$ the condition $H^{1}(\mathcal{O}(-4) \otimes \mathcal{O}(t+6-(3+$ $2)))=0$ is satisfied for every $t \geq 0$, but $H^{0}(\mathcal{O}(-4) \otimes \mathcal{O}(k))=H^{0}(\mathcal{O}(2)) \neq 0$. So $\mathcal{F}(1)=\mathcal{O}(-4)$ is wregular.

Now we show how weighted regularity and weighted semiregularity behave under pullback along the natural covering map from $\mathbb{P}^{n}$. 
Lemma 2.8. Let $\mathcal{F}$ be an $m$-semiwregular (or $m$-wregular) coherent sheaf on $\mathbb{P}$. Then $\pi^{*} \mathcal{F}$ is $\left((m+1) k-n+\mathbf{w}-\mathbf{w}_{1}\right)$-regular on $\mathbb{P}^{n}$.

Proof. We want to show that, for $q=(m+1) k-n+\mathbf{w}-\mathbf{w}_{1}$ and for any $i=1, \ldots, n$, we have

$$
h^{i}\left(\mathbb{P}^{n}, \pi^{*} \mathcal{F}(q-i)\right)=0 .
$$

By (3), $\pi_{*} \pi^{*} \mathcal{F} \cong \bigoplus_{\left(r_{0}, \ldots, r_{n}\right)} \mathcal{F}(-\mathbf{r})$, where $0 \leq r_{j}<w_{j}$ for all $j=0, \ldots, n$. The smallest twist that occurs is $-\mathbf{w}+n+1$ (when $r_{j}=w_{j}-1$ for every j). Since

$$
H^{i}\left(\mathbb{P}^{n}, \pi^{*} \mathcal{F}(q-i)\right) \cong H^{i}\left(\mathbb{P}, \pi_{*} \pi^{*} \mathcal{F}(q-i)\right)
$$

it is enough to show that $H^{i}(\mathbb{P}, \mathcal{F}(q-i-\mathbf{w}+n+1))$ vanishes, for each $i$, with $q$ as above.

If $i=1$ and $q=(m+1) k-n+\mathbf{w}-\mathbf{w}_{1}$ we get

$$
H^{1}(\mathbb{P}, \mathcal{F}(q-1-\mathbf{w}+n+1))=H^{1}\left(\mathcal{F}\left((m+1) k-\mathbf{w}_{1}\right)\right)
$$

which is zero because $\mathcal{F}$ is $m$-wregular. Hence

$$
H^{1}\left(\mathbb{P}, \pi_{*} \pi^{*} \mathcal{F}\left((m+1) k-n+\mathbf{w}-\mathbf{w}_{1}-1\right)\right)=0 .
$$

For $2 \leq i \leq n$ we have

$$
H_{*}^{i}\left(\mathbb{P}, \mathcal{F}\left(t+(m+1) k-\mathbf{w}_{i}\right)\right)=0
$$

for every $t \geq 0$, by $m$-wregularity. However, $\mathbf{w}_{i} \geq \mathbf{w}_{1}+i-1$, since the weights are positive integers, so

$$
\begin{aligned}
q-i-\mathbf{w}+n+1 & =(m+1) k-n+\mathbf{w}-\mathbf{w}_{1}-i-\mathbf{w}+n+1 \\
& =(m+1) k-\mathbf{w}_{1}-i+1 \\
& \geq m(k+1)-\mathbf{w}_{i}
\end{aligned}
$$

and hence $H^{i}(\mathbb{P}, \mathcal{F}(q-i-\mathbf{w}+n+1))=0$, as required.

Next we show how weighted semiregularity behaves under restriction to coordinate hyperplanes. Weighted regularity does not behave well, in general, as Example 2.10 illustrates.

Lemma 2.9. Suppose that $\mathcal{F}$ is an m-semiwregular coherent sheaf on $\mathbb{P}$ and let $\mathbb{H}_{j}=\left\{x_{j}=0\right\}$ be the $j$-th coordinate hyperplane. We put $k_{j}=$ $\operatorname{lcm}\left(w_{0}, \ldots, w_{j-1}, w_{j+1}, \ldots, w_{n}\right)$ and $z_{j}=k / k_{j}$. Then $\mathcal{F}_{\mathbb{H}_{j}}=\mathcal{F} \otimes \mathcal{I}_{\mathbb{H}_{j}}$ is $\left((m+1) z_{j}-1\right)$-semiwregular on $\mathbb{H}_{j}$.

Proof. We consider $\mathbb{H}_{j} \cong \mathbb{P}\left(w_{0}, \ldots, w_{j-1}, w_{j+1}, \ldots, w_{n}\right)$ : note that the sum of the first $i+1$ of these weights is $\mathbf{w}_{i}$ if $i<j$ and is $\mathbf{w}_{i+1}-w_{j}$ if $i \geq j$. 
Thus, writing $\mathcal{E}=\mathcal{F}_{\mathbb{H}_{j}}$, we want to show that, for $q=(m+1) z_{j}-1$ and for any $i=1, \ldots, n-1$

$$
\begin{aligned}
& H^{i}\left(\mathbb{H}_{j}, \mathcal{E}\left(t+(q+1) k_{j}-\mathbf{w}_{i}\right)\right)=0 \quad \text { if } i<j \\
& H^{i}\left(\mathbb{H}_{j}, \mathcal{E}\left(t+(q+1) k_{j}-\left(\mathbf{w}_{i+1}-w_{j}\right)\right)\right)=0 \quad \text { if } i \geq j,
\end{aligned}
$$

for every integer $t \geq 0$.

Let us consider the exact sequence

$$
0 \longrightarrow \mathcal{F}\left(-w_{j}\right) \longrightarrow \mathcal{F} \longrightarrow \mathcal{E} \longrightarrow 0,
$$

coming from tensoring $\mathcal{F}$ with the restriction sequence

$$
0 \longrightarrow \mathcal{O}_{\mathbb{P}}\left(-w_{j}\right) \longrightarrow \mathcal{O}_{\mathbb{P}} \longrightarrow \mathcal{O}_{\mathbb{H}_{j}} \longrightarrow 0 .
$$

If $i<j$ we twist (7) by $t+(m+1) k-\mathbf{w}_{i}$ : in cohomology, this gives (for $0<i<n$ and $t \geq 0$ )

$$
\begin{aligned}
H^{i}\left(\mathbb{P}, \mathcal{F}\left(t+(m+1) k-\mathbf{w}_{i}\right)\right) & \longrightarrow H^{i}\left(\mathbb{H}_{j}, \mathcal{E}\left(t+(m+1) k-\mathbf{w}_{i}\right)\right) \\
& \longrightarrow H^{i+1}\left(\mathbb{P}, \mathcal{F}\left(t+(m+1) k-\mathbf{w}_{i}-w_{j}\right)\right) .
\end{aligned}
$$

The first of these terms vanishes because $\mathcal{F}$ is $m$-wregular, and the $m$ wregularity also gives $H^{i+1}\left(\mathbb{P}, \mathcal{F}\left(t^{\prime}+(m+1) k-\mathbf{w}_{i+1}\right)=0\right.$ for any $t^{\prime} \geq 0$. In particular, since $i+1 \leq j$ we have $w_{i+1} \geq w_{j}$ and we may take $t^{\prime}=$ $t+w_{i+1}-w_{j}$, giving us vanishing of the third term in (8). Thus the middle term also vanishes, and since $(q+1) k_{j}=(m+1) k$ that proves (5).

The proof for the second case, $i \geq j$, is similar. This time we twist (7) by $t+(m+1) k-\left(\mathbf{w}_{i+1}-w_{j}\right)$. In cohomology this gives

$$
\begin{array}{r}
H^{i}\left(\mathbb{P}, \mathcal{F}\left(t+(m+1) k-\left(\mathbf{w}_{i+1}-w_{j}\right)\right) \longrightarrow H^{i}\left(\mathbb{H}_{j}, \mathcal{E}\left(t+(m+1) k-\left(\mathbf{w}_{i+1}-w_{j}\right)\right)\right)\right. \\
\longrightarrow H^{i+1}\left(\mathbb{P}, \mathcal{F}\left(t+(m+1) k-\mathbf{w}_{i+1}\right)\right) .
\end{array}
$$

The third of these terms vanishes because $\mathcal{F}$ is $m$-wregular, and the $m$ wregularity also gives $H^{i}\left(\mathbb{P}, \mathcal{F}\left(t^{\prime}+(m+1) k-\mathbf{w}_{i}\right)=0\right.$ for any $t^{\prime}>0$. Now since $i+1 \geq j$ we have $w_{i+1} \leq w_{j}$ and we may take $t^{\prime}=t-w_{i+1}+w_{j}$, giving us vanishing of the first term in (9). Again, the middle term also vanishes and this proves (6).

Example 2.10. If $\mathbb{P}=\mathbb{P}(3,2,1)$, then $z_{2}=1$, it is easy to check that $\mathcal{F}=$ $\mathcal{O}_{\mathbb{P}}(-5)$ is 0-wregular. In fact, $k=6$, so $H^{2}(\mathcal{O}(-5+6-6)) \cong H^{0}(\mathcal{O}(-1))=$ 0 , and thus if we take $\mathcal{F}=\mathcal{O}(-5)$ then the condition $H^{2}(\mathcal{O}(-5) \otimes \mathcal{O}(t+$ $6-(3+2+1)))=0$ is satisfied for every $t \geq 0$, and $H^{0}(\mathcal{O}(-5) \otimes \mathcal{O}(k))=$ $H^{0}(\mathcal{O}(1)) \neq 0$. However $\mathcal{F}_{\mathbb{H}_{2}}$ is not 0 -wregular by Example 2.7.

Remark 2.11. Let $\mathcal{F}$ be an $m$-wregular coherent sheaf on $\mathbb{P}$. If $w_{0}=\cdots=$ $w_{n}=1$, then $(m+1) k-n+\mathbf{w}_{n-2}=m$, and $\pi^{*} \mathcal{F}$ is m-regular. More generally, if $w_{j}=1$, then $z_{j}(m+1)-1=m$ and $\mathcal{F}_{\mathbb{H}_{j}}$ is $m$-semiwregular on $\mathbb{H}_{j}$. 
We cannot expect the above properties for toric regularity.

We can give a notion of global generation adapted to this weighted situation.

Definition 2.12. A coherent sheaf $\mathcal{F}$ on $\mathbb{P}$ is said to be weighted globally generated (abbreviated to wgg) if, for any $x \in \mathbb{P}$, the map

$$
\mu: \bigoplus_{j=0}^{n} H^{0}\left(\mathcal{F}\left(k-w_{j}\right)\right) \otimes \mathcal{O}_{x} \rightarrow \mathcal{F}_{x}(k),
$$

where $\mu\left(\sum_{j=0}^{n} f_{j} \otimes e_{x}\right)=\sum_{j=0}^{n} f_{j} x_{j} e_{x}$, is surjective.

This reduces to the usual definition of globally generated in the case of $\mathbb{P}^{n}$, when $w_{0}=\cdots=w_{n}=1$ and $k=1$, so $\bigoplus_{j=0}^{n} H^{0}\left(\mathcal{F}\left(k-w_{j}\right)\right) \cong$ $H^{0}(\mathcal{F}) \otimes H^{0}(\mathcal{O}(1))$. In fact we have a surjection

$$
\mu_{x}: H^{0}(\mathcal{F}) \otimes H^{0}(\mathcal{O}(1)) \otimes \mathcal{O}_{x} \rightarrow \mathcal{F}_{x}(1)
$$

and a surjection

$$
H^{0}(\mathcal{F}) \otimes H^{0}(\mathcal{O}(1)) \otimes \mathcal{O}_{x} \rightarrow H^{0}(\mathcal{F}) \otimes \mathcal{O}_{x}(1) .
$$

So we may construct a surjection

$$
H^{0}(\mathcal{F}) \otimes \mathcal{O}_{x}(1) \rightarrow \mathcal{F}_{x}(1) .
$$

Finally we may identify $\mathcal{O}_{\mathbb{P}}(1)$ with $\mathcal{O}_{\mathbb{P}}$ at $x$ and $\mathcal{F}(1)$ with $\mathcal{F}$ at $x$. So we may conclude that $\mathcal{F}$ is globally generated.

Proposition 2.13. $\mathcal{O}_{\mathbb{P}}$ is wgg.

Proof. We want to show that for any $i=0, \ldots, n$ the map

$$
\mu_{i}: \bigoplus_{j=0}^{n} H^{0}\left(\mathcal{O}\left(k-w_{j}\right)\right) \otimes \Gamma\left(D_{+}\left(x_{i}\right), \mathcal{O}\right) \rightarrow \Gamma\left(D_{+}\left(x_{i}\right), \mathcal{O}(k)\right)
$$

is surjective, where $D_{+}\left(x_{i}\right)$ denotes as usual the locus $\left(x_{i} \neq 0\right)$.

Let $u \in \Gamma\left(D_{+}\left(x_{i}\right), \mathcal{O}(k)\right)$; then $u=a x_{i}^{-s}$ with $s>0$ and $a$ a monomial of degree $s w_{i}+k$. Therefore $u=a x_{i}^{s k-s} / x_{i}^{s k}$ and

$$
\operatorname{deg}\left(a x_{i}^{s k-s}\right)=s w_{i}+k+w_{i}(s k-s)=w_{i} s k+k=k\left(w_{i} s+1\right) .
$$

So $a x_{i}^{s k-s}$ is a monomial containing $x_{i}^{s k-s}$ and its degree is a multiple of $k$. This means that we can write $a x_{i}^{s k-s}=a^{\prime} b$ where $a^{\prime}=x_{i}^{k / w_{i}-1}$ and has degree $k-w_{i}$; then $b / x_{i}^{s k}$ has degree $k\left(s w_{i}+1\right)-k+w_{i}-s k w_{i}=w_{i}$ so the map

$$
H^{0}\left(\mathcal{O}\left(k-w_{i}\right)\right) \otimes \Gamma\left(D_{+}\left(x_{i}\right), \mathcal{O}\left(w_{i}\right)\right) \longrightarrow \Gamma\left(D_{+}\left(x_{i}\right), \mathcal{O}(k)\right)
$$

is surjective.

Finally let us notice that $\Gamma\left(D_{+}\left(x_{i}\right), \mathcal{O}\left(w_{i}\right)\right) \cong \Gamma\left(D_{+}\left(x_{i}\right), \mathcal{O}\right)$.

This means that the map $\mu_{i}$ is surjective for any $i=0, \ldots n$, and hence the map $\mu$ of Definition 2.12 is surjective at every point $x \in \mathbb{P}$. 
In general, $\mathcal{O}_{\mathbb{P}}(m)$ is not globally generated in the usual sense: see for example [5, Theorem 4B.7]. On the other hand we have the following proposition.

Proposition 2.14. If $m>0$ and $\mathcal{O}_{\mathbb{P}}(m)$ is globally generated then $\mathcal{O}_{\mathbb{P}}(m)$ is wgg.

Proof. We want to show that for any $i=0, \ldots, n$ the map

$$
\mu_{i}: \bigoplus_{j=0}^{n} H^{0}\left(\mathcal{O}\left(k+m-w_{j}\right)\right) \otimes \Gamma\left(D_{+}\left(x_{i}\right), \mathcal{O}\right) \longrightarrow \Gamma\left(D_{+}\left(x_{i}\right), \mathcal{O}(m+k)\right)
$$

is surjective.

Let $u \in \Gamma\left(D_{+}\left(x_{i}\right), \mathcal{O}(m)\right)$ : then $u=a x_{i}^{-s}$ with $s>0$ and $a$ a monomial of degree $s w_{i}+m$. Therefore $u=a x_{i}^{s k-s} / x_{i}^{s k}$ and

$$
\operatorname{deg}\left(a x_{i}^{s k-s}\right)=s w_{i}+m+w_{i}(s k-s)=w_{i} s k+m
$$

Now since $\mathcal{O}(m)$ is globally generated we can write $a x_{i}^{s k-s}=a^{\prime} b$, where $a^{\prime}$ has degree $m$ and $b / x_{i}^{s k}$ has degree 0 . This means that we can write $a^{\prime} b=a^{\prime \prime} b^{\prime}$ where $a^{\prime \prime}=a^{\prime} x_{i}^{-1}$ and has degree $m-w_{i}$, and $b^{\prime}=b x_{i}$ so that $b^{\prime} / x_{i}^{s k}$ has degree $w_{i}$. In this way we have that the map

$$
H^{0}\left(\mathcal{O}\left(k+m-w_{i}\right)\right) \otimes \Gamma\left(D_{+}\left(x_{i}\right), \mathcal{O}\left(w_{i}\right)\right) \longrightarrow \Gamma\left(D_{+}\left(x_{i}\right), \mathcal{O}(m+k)\right)
$$

is surjective.

Now we prove the analogues for weighted regularity of the main properties of Castelnuovo-Mumford regularity.

Theorem 2.15. Let $\mathcal{F}$ be a wregular coherent sheaf on $\mathbb{P}$.

(i) $H^{0}(\mathcal{F}(k))$ is spanned by $H^{0}\left(\mathcal{F}\left(k-w_{0}\right)\right) \oplus \cdots \oplus H^{0}\left(\mathcal{F}\left(k-w_{n}\right)\right)$.

(ii) $\mathcal{F}$ is $m$-wregular for all $m \geq 0$.

(iii) $\mathcal{F}$ is wgg.

Proof. (i) is clear from the Koszul sequence (44) twisted by $\mathcal{F}(k)$. Moreover since $H^{0}(\mathcal{F}(k)) \neq 0$ the surjection is non-trivial.

(ii) is clear by the definition of wregularity.

(iii) we prove as follows. Choose $l \in k \mathbb{Z}$ so that $\mathcal{F}(k+l)$ and $\mathcal{O}(l)$ are globally generated, which holds for $l \gg 0$, and consider the (not exact!) sequence

$$
\begin{aligned}
\bigoplus_{j} H^{0}\left(\mathcal{F}\left(k-w_{j}\right)\right) \otimes H^{0}(\mathcal{O}(l)) \otimes & \mathcal{O} \stackrel{\mu}{\longrightarrow} H^{0}(\mathcal{F}(k)) \otimes H^{0}(\mathcal{O}(l)) \otimes \mathcal{O} \\
& \stackrel{\mu^{\prime}}{\longrightarrow} H^{0}(\mathcal{F}(k+l)) \otimes \mathcal{O} \stackrel{\mu^{\prime \prime}}{\longrightarrow} \mathcal{F}(k+l) .
\end{aligned}
$$


Notice that $\mu$ is non-trivial and surjective by (i), and $\mu^{\prime}$ and $\mu^{\prime \prime}$ are both surjective because $\mathcal{F}(k+l)$ and $\mathcal{O}(l)$ are globally generated. Near a point $x$, fix an isomorphism between $\mathcal{O}(l)$ and $\mathcal{O}$ : this identifies $\mathcal{O}(l)$ with $\mathcal{O}$ and $\mathcal{F}(k)_{x}$ with $\mathcal{F}(k+l)_{x}$. Then $H^{0}(\mathcal{O}(l))$ becomes just a vector space of elements of the local ring $\mathcal{O}_{x}$, so we have that $\mathcal{F}(k)$ is wgg.

\section{Monads on weighted projective spaces}

In this section we assume that $n=\operatorname{dim} \mathbb{P} \geq 3$. We begin with a preliminary definition.

Definition 3.1. Suppose that $\mathcal{E}$ and $\mathcal{E}^{\prime}$ are vector bundles on a projective variety $X$. A surjective map $\eta: \mathcal{E} \rightarrow \mathcal{E}^{\prime}$ is said to be minimal if no rank 1 direct summand of $\mathcal{E}^{\prime}$ is the image of a line subbundle of $\mathcal{E}$.

Next we recall the basic definitions about monads, due to Horrocks [10].

Definition 3.2. A sequence of bundles on a projective variety $X$

$$
\mathcal{A} \stackrel{\alpha}{\longrightarrow} \mathcal{B} \stackrel{\beta}{\longrightarrow} \mathcal{C}
$$

such that $\mathcal{A}$ and $\mathcal{C}$ are sums of line bundles, $\alpha$ is injective, $\beta$ is surjective and $\beta \alpha=0$ is called a monad on $X$.

The vector bundle $\mathcal{E}=\frac{\mathrm{ker} \beta}{\operatorname{im} \alpha}$ is called the homology of the monad.

$A$ monad is said to be minimal if the maps $\alpha^{\vee}: \mathcal{B}^{\vee} \rightarrow \mathcal{A}^{\vee}$ and $\beta: \mathcal{B} \rightarrow \mathcal{C}$ are minimal.

In particular if $\mathcal{B}$ is a sum of line bundles, the maps $\alpha$ and $\beta$ are just matrices and then minimal means that no matrix entry is a non-zero scalar both in $\alpha$ and in $\beta$.

Horrocks showed in [10] that every bundle $\mathcal{E}$ on $\mathbb{P}^{n}$ with $n \geq 3$ is the homology of a minimal monad. Now we extend this correspondence to $\mathbb{P}$ : we generalize the proof of Proposition 3 in [4]. First we need a definition (see equation (11) in Section 1 for the notation).

Definition 3.3. For $l \in \mathbb{Z}$, a minimal $l$-resolution of a bundle $\mathcal{E}$ is an exact sequence

$$
0 \longrightarrow \mathcal{E} \longrightarrow \mathcal{P} \stackrel{\pi}{\longrightarrow} \mathcal{C} \longrightarrow 0
$$

in which $\mathcal{C}$ splits, $\pi$ is minimal and $H^{1}(\mathcal{P}(\geq l))=0$.

Theorem 3.4. Every bundle $\mathcal{E}$ on $\mathbb{P}$ is the homology of a minimal monad with $\mathcal{B}$ satisfying

(i) $H_{*}^{1}(\mathcal{B})=H_{*}^{n-1}(\mathcal{B})=0$

(ii) $H_{*}^{i}(\mathcal{B})=H_{*}^{i}(\mathcal{E})$ if $1<i<n-1$. 
Proof. The module $H_{*}^{i}(\mathcal{E})$ has finite length for $0<i<n$, because $\mathbb{P}$ is arithmetically Cohen-Macaulay and subcanonical, and for any $t \in \mathbb{Z}$ we have

$$
H^{i}(X, \mathcal{E}(t)) \cong H^{n-i}\left(X, \mathcal{E}^{\vee}(-t+e)\right)^{\vee} .
$$

We start by proving that every bundle $\mathcal{E}$ has a minimal $l$-resolution for each $l \in \mathbb{Z}$. Consider the module $H^{1}(\mathcal{E}(\geq l))$ and a minimal system of generators $g_{1}, \ldots, g_{r}$. For each $i \geq l$, write $q_{i}$ for the number of generators in degree $i$ : since the module has finite length there is an $l_{0}$ such that $q_{i}=0$ if $i>l_{0}$. So our system $g_{1}, \ldots, g_{r}$ is an element of

$$
q_{l} H^{1}(\mathcal{E}(l)) \oplus \cdots \oplus q_{l_{0}} H^{1}\left(\mathcal{E}\left(l_{0}\right)\right) \cong H^{1}\left(\mathcal{E} \otimes \mathcal{C}^{\vee}\right)
$$

where $\mathcal{C}=q_{l} \mathcal{O}(-l) \oplus \cdots \oplus q_{l_{0}} \mathcal{O}\left(-l_{0}\right)$. But, since $H^{1}\left(\mathcal{E} \otimes \mathcal{C}^{\vee}\right) \cong \operatorname{Ext}^{1}(\mathcal{C}, \mathcal{E})$ we have $\left\{g_{i}\right\} \in \operatorname{Ext}^{1}(\mathcal{C}, \mathcal{E})$, so we can associate an extension

$$
0 \longrightarrow \mathcal{E} \longrightarrow \mathcal{P} \longrightarrow \mathcal{C} \longrightarrow 0
$$

to our system of generators.

Now, looking at the sequence in cohomology we see that the map

$$
f: H^{0}(\mathcal{C}(\geq l)) \longrightarrow H^{1}((\mathcal{E}(\geq l))
$$

is surjective by construction. Moreover, since $\mathbb{P}$ is $\mathrm{ACM}$, all the intermediate cohomology of $\mathcal{C}$ vanishes and we can conclude that $H^{1}(\mathcal{P}(\geq l))=0$. Thus the sequence (10) is an $l$-resolution, and it is minimal because the system $\left\{g_{i}\right\}$ was chosen minimal.

This $l$-resolution will be the last column of our display.

In the same way, for every $l^{\prime} \in \mathbb{Z}$, we can find an $l^{\prime}$-resolution

$$
0 \longrightarrow \mathcal{E}^{\vee} \longrightarrow \mathcal{Q}^{\vee} \longrightarrow \mathcal{A}^{\vee} \longrightarrow 0
$$

for $\mathcal{E}^{\vee}$ and the dual of (11) will be the first row, so we have

$$
\begin{aligned}
& \\
& 0 \\
& \downarrow \\
& \mathcal{E} \\
& \downarrow \\
& \mathcal{P} \\
& \downarrow \\
& \mathcal{C} \\
& \downarrow \\
& 0
\end{aligned}
$$

where $\mathcal{A}=\bigoplus_{i} \mathcal{O}\left(a_{i}\right)$ is a bundle without intermediate cohomology, with $a_{i} \geq l^{\prime}$ for all $i$. Moreover

$$
H^{n-1}\left(\mathcal{Q}\left(\leq-l^{\prime}+e\right)\right) \cong H^{1}\left(\mathcal{Q}^{\vee}\left(\geq l^{\prime}\right)\right)=0 .
$$


We observe that if $n \geq 3$

$$
\operatorname{Ext}^{i}(\mathcal{C}, \mathcal{A})=H^{i}\left(\mathcal{C}^{\vee} \otimes \mathcal{A}\right)=0
$$

for $i=1,2$. Then applying the functor $\operatorname{Hom}(\bullet, \mathcal{A})$ to (10) we have

$$
0=\operatorname{Ext}^{1}(\mathcal{C}, \mathcal{A}) \rightarrow \operatorname{Ext}^{1}(\mathcal{P}, \mathcal{A}) \rightarrow \operatorname{Ext}^{1}(\mathcal{E}, \mathcal{A}) \rightarrow \operatorname{Ext}^{2}(\mathcal{C}, \mathcal{A})=0,
$$

so

$$
\operatorname{Ext}^{1}(\mathcal{P}, \mathcal{A}) \cong \operatorname{Ext}^{1}(\mathcal{E}, \mathcal{A})
$$

This means that the extension in our row (the dual of (11)) comes from the unique extension

$$
0 \longrightarrow \mathcal{A} \stackrel{\alpha}{\longrightarrow} \mathcal{B} \longrightarrow \mathcal{P} \longrightarrow 0
$$

and we have

$$
\begin{aligned}
& \begin{array}{rll}
0 & \\
& \downarrow \\
\downarrow & \downarrow \\
\mathcal{A} & \downarrow \\
\mathcal{Q} & \mathcal{E}
\end{array} \rightarrow 0 \\
& 0 \rightarrow \mathcal{A} \stackrel{\alpha}{\rightarrow} \stackrel{\downarrow}{\mathcal{B}} \rightarrow \stackrel{\downarrow}{\mathcal{P}} \rightarrow 0 . \\
& \stackrel{\downarrow}{\mathcal{C}}=\stackrel{\downarrow}{\mathcal{C}} \\
& \downarrow \quad \downarrow
\end{aligned}
$$

This is the display of the monad

$$
\mathcal{A} \stackrel{\alpha}{\longrightarrow} \mathcal{B} \stackrel{\beta}{\longrightarrow} \mathcal{C} .
$$

The minimality comes from the minimality of the two resolutions. From the first row we see that $H_{*}^{i}(\mathcal{E}) \cong H_{*}^{i}(\mathcal{Q})$ for $0<i<n-1$. Looking at the first column in cohomology,

$0=H^{n-1}\left(\mathcal{Q}\left(\leq-l^{\prime}+e\right)\right) \rightarrow H^{n-1}\left(\mathcal{B}\left(\leq-l^{\prime}+e\right)\right) \rightarrow H^{n-1}\left(\mathcal{C}\left(\leq-l^{\prime}+e\right)\right)=0$

we have that $H^{n-1}\left(\mathcal{B}\left(\leq-l^{\prime}+e\right)\right)=0$, and $H_{*}^{i}(\mathcal{E}) \cong H_{*}^{i}(\mathcal{Q}) \cong H_{*}^{i}(\mathcal{B})$ for $1<i<n-1$.

Looking at the second row in cohomology,

$$
0=H^{1}(\mathcal{A}(\geq l)) \rightarrow H^{n-1}(\mathcal{B}(\geq l)) \rightarrow H^{n-1}(\mathcal{C}(\geq l))=0,
$$

we see that $H^{1}(\mathcal{B}(\geq l))=0$. If we choose $l$ and $l^{\prime}$ small enough we get the claimed conditions (i) and (ii) above:

(i) $H_{*}^{1}(\mathcal{B})=H_{*}^{n-1}(\mathcal{B})=0$

(ii) $H_{*}^{i}(\mathcal{B})=H_{*}^{i}(\mathcal{E})$ for $1<i<n-1$. 
If the bundles in the monad all split, we can get some results about wregularity for $\mathcal{E}$.

Definition 3.5. A monad on $\mathbb{P}$ is called quasi-linear if it has the form

$$
\bigoplus_{i=1}^{s} \mathcal{O}_{\mathbb{P}}\left(a_{i}\right) \stackrel{\alpha}{\longleftrightarrow} \bigoplus_{l=1}^{r+s+t} \mathcal{O}_{\mathbb{P}}\left(b_{l}\right) \stackrel{\beta}{\longrightarrow} \bigoplus_{j=1}^{t} \mathcal{O}_{\mathbb{P}}\left(c_{j}\right) .
$$

By convention, we shall write the twists in increasing order, $a_{i} \leq a_{i+1}$, etc.: note that this is the opposite of our convention for the weights.

We prove an analogue of [8, Theorem 3.2].

Theorem 3.6. Let $\mathcal{E}$ be a rank $r$ vector bundle on $\mathbb{P}$ which is the homology of a quasi-linear monad (12). Put $c=\sum_{j=1}^{t} c_{t}$. Then $\mathcal{E}$ is $m$-wregular for any integer $m$ such that $H^{0}(\mathcal{E}((m+1) k)) \neq 0$ and

$(m+1) k \geq \max \left\{(n-1) c_{t}-\left(b_{1}+\cdots+b_{t+n}\right)-\left(\mathbf{w}-\mathbf{w}_{1}\right)+1+c,-b_{1}+1,-a_{1}+1\right\}$.

Proof. Let us consider the short exact sequences from the display of the monad:

$$
0 \rightarrow \mathcal{K} \rightarrow \bigoplus_{l=1}^{r+s+t} \mathcal{O}_{\mathbb{P}}\left(b_{l}\right) \stackrel{\beta}{\rightarrow} \bigoplus_{j=1}^{t} \mathcal{O}_{\mathbb{P}}\left(c_{j}\right) \rightarrow 0
$$

and

$$
0 \rightarrow \bigoplus_{i=1}^{s} \mathcal{O}_{\mathbb{P}}\left(a_{i}\right) \rightarrow \mathcal{K} \rightarrow \mathcal{E} \rightarrow 0 .
$$

We get $H^{i}(\mathcal{K}(p))=H^{i}(\mathcal{E}(p))=0$ for any integer $p$ and any $i=2, \ldots, n-2$. Moreover if $p \geq \max \left\{-b_{1}-\mathbf{w}+1,-a_{1}-\mathbf{w}+1\right\}$ we have also $H^{i}(\mathcal{K}(p))=$ $H^{i}(\mathcal{E}(p))=0$ for $i \geq n-1$. So if $(m+1) k \geq \max \left\{-b_{1}+1,-a_{1}+1\right\}$ we may conclude that

$$
H^{n}(\mathcal{E}((m+1) k-\mathbf{w})=0 .
$$

To see which are the $p$ for which $H^{1}(\mathcal{K}(p)) \cong H^{1}(\mathcal{E}(p))=0$, we consider the Buchsbaum-Rim complex associated to

$$
\mathcal{F}=\bigoplus_{l=1}^{r+s+t} \mathcal{O}_{\mathbb{P}}\left(b_{l}\right) \stackrel{\beta}{\longrightarrow} \mathcal{G}=\bigoplus_{j=1}^{t} \mathcal{O}_{\mathbb{P}}\left(c_{j}\right),
$$

which is the complex

$$
\begin{aligned}
S^{r+s-1} \mathcal{G}^{\vee} & \otimes \wedge^{r+s+t} \mathcal{F} \rightarrow S^{r+s-2} \mathcal{G}^{\vee} \otimes \wedge^{r+s+t-1} \mathcal{F} \rightarrow \cdots \rightarrow S^{2} \mathcal{G}^{\vee} \otimes \wedge^{3+t} \mathcal{F} \\
& \rightarrow \mathcal{G}^{\vee} \otimes \wedge^{2+t} \mathcal{F} \rightarrow \wedge^{1+t} \mathcal{F} \rightarrow \mathcal{F} \otimes \mathcal{O}_{\mathbb{P}}(c) \rightarrow \mathcal{G} \otimes \mathcal{O}_{\mathbb{P}}(c) \rightarrow 0 .
\end{aligned}
$$


We cut (14) into short exact sequences

$$
\begin{aligned}
& 0 \rightarrow \mathcal{K} \otimes \mathcal{O}_{\mathbb{P}}(c) \rightarrow \mathcal{F} \otimes \mathcal{O}_{\mathbb{P}}(c) \rightarrow \mathcal{G} \otimes \mathcal{O}_{\mathbb{P}}(c) \rightarrow 0, \\
& 0 \rightarrow \mathcal{K}_{2} \rightarrow \wedge^{1+t} \mathcal{F} \rightarrow \mathcal{K} \otimes \mathcal{O}_{\mathbb{P}}(c) \rightarrow 0, \\
& 0 \rightarrow \mathcal{K}_{3} \rightarrow \mathcal{G}^{\vee} \otimes \wedge^{2+t} \mathcal{F} \rightarrow \mathcal{K}_{2} \rightarrow 0 \\
& \quad \vdots \\
& 0 \rightarrow \mathcal{K}_{n} \rightarrow S^{n-2} \mathcal{G}^{\vee} \otimes \wedge^{t+n-1} \mathcal{F} \rightarrow \mathcal{K}_{n-1} \rightarrow 0, \\
& 0 \rightarrow \mathcal{K}_{n+1} \rightarrow S^{n-1} \mathcal{G}^{\vee} \otimes \wedge^{t+n} \mathcal{F} \rightarrow \mathcal{K}_{n} \rightarrow 0 .
\end{aligned}
$$

Note that

$$
S^{n-1} \mathcal{G}^{\vee} \otimes \wedge^{t+n} \mathcal{F}=\bigoplus_{q} \mathcal{O}_{\mathbb{P}}\left(d_{q}\right)
$$

where $d_{q}=\left(b_{l_{1}}+\cdots+b_{l_{t+n}}\right)-\left(c_{j_{1}}+\cdots+c_{j_{n-1}}\right)$ with $l_{1}<\cdots<l_{t+n}$ and $j_{1} \leq \cdots \leq j_{n-1}$. Now from the cohomological exact sequences associated to the above short exact sequences tensored by $\mathcal{O}_{\mathbb{P}}(p-c)$ we get

$$
\begin{aligned}
h^{1}(\mathcal{K}(p))=h^{2}\left(\mathcal{K}_{2}(p-c)\right)=\cdots & =h^{n}\left(\mathcal{K}_{n}(p-c)\right) \\
& \leq h^{n}\left(S^{n-1} \mathcal{G}^{\vee} \otimes \wedge^{t+n} \mathcal{F} \otimes \mathcal{O}_{\mathbb{P}}(p-c)\right)
\end{aligned}
$$

which is zero if $p \geq(n-1) c_{t}-\left(b_{1}+\cdots+b_{t+n}\right)-\mathbf{w}+1+c$. In fact, since

$$
(n-1) c_{t}-\left(c_{j_{1}}+\cdots+c_{j_{n-1}}\right) \geq 0
$$

and

$$
\left(b_{l_{1}}+\cdots+b_{l_{t+n}}\right)-\left(b_{1}+\cdots+b_{t+n}\right) \geq 0,
$$

we have $d_{q}+p-c \geq-\mathbf{w}$. So we get

$$
H^{1}\left(\mathcal{E}\left((m+1) k-\left(w_{n-1}+w_{n}\right)\right)\right)=0
$$

if $(m+1) k \geq(n-1) c_{t}-\left(b_{1}+\cdots+b_{t+n}\right)-\left(w_{0}+\cdots+w_{n-2}\right)+1+c$.

Remark 3.7. In the case of $\mathbb{P}=\mathbb{P}^{n}$ the bound (13) reduces to $m+1 \geq \max \left\{(n-1) c_{t}-\left(b_{1}+\cdots+b_{t+n}\right)-(n-1)+1+c,-b_{1}+1,-a_{1}+1\right\}$ which is precisely the bound of [8, Theorem 3.2]

Finally we want to discuss the sharpness of the bound in Theorem 3.6 .

Example 3.8. Take $\mathbb{P}=\mathbb{P}(3,2,2,1)$ and consider the bundle $\mathcal{E}$ given by the monad

$$
\mathcal{O}_{\mathbb{P}}(-2) \stackrel{\alpha}{\longleftrightarrow} \mathcal{O}_{\mathbb{P}}(-1) \oplus \mathcal{O}_{\mathbb{P}}^{\oplus 2} \oplus \mathcal{O}_{\mathbb{P}}(1) \stackrel{\alpha^{\vee}}{\longrightarrow} \mathcal{O}_{\mathbb{P}}(2),
$$

where $\alpha^{\vee}=\left(x_{0}, x_{1}, x_{2}, x_{3}\right)$. In this case the bound given by (13) is sharp. 
In fact, we have $k=3, a_{1}=-2, b_{1}=-1, b_{2}=b_{3}=0, b_{4}=1$ and $c_{t}=c=c_{1}=2$, so we get

$$
(m+1) 3 \geq \max \{(2) 2-(0)-(3)+1+2,1+1,2+1\}=4,
$$

so $m=1$. On the other hand we notice that $\mathcal{E}$ is not wregular (i.e. we cannot take $m=0$ ), so the bound is sharp. In fact from the sequences

$$
0 \rightarrow \mathcal{K} \rightarrow \mathcal{O}_{\mathbb{P}}(-1) \oplus \mathcal{O}_{\mathbb{P}}^{\oplus 2} \oplus \mathcal{O}_{\mathbb{P}}(1) \rightarrow \mathcal{O}_{\mathbb{P}}(2) \rightarrow 0
$$

and

$$
0 \rightarrow \mathcal{O}_{\mathbb{P}}(-2) \rightarrow \mathcal{K} \rightarrow E \rightarrow 0
$$

we get $H^{3}(\mathcal{E}(3-8)) \neq 0$.

\section{References}

[1] E. Arrondo, F. Malaspina, Cohomological characterization of vector bundles on Grassmannians of lines, J. Algebra 323 (2010), no. 4, 1098-1106.

[2] E. Ballico, F. Malaspina, Qregularity and an extension of the Evans-Griffiths criterion to vector bundles on quadrics, J. Pure Appl. Algebra 213 (2009), 194-202.

[3] E. Ballico, F. Malaspina, Regularity and cohomological splitting conditions for vector bundles on multiprojective spaces, J. Algebra 345 (2011), 137-149.

[4] W. Barth, K. Hulek, Monads and moduli of vector bundles, Manuscripta Math. 25 (1978), 323-447.

[5] M. Beltrametti and L. Robbiano, Introduction to the theory of weighted projective spaces, Exposition. Math. 4 (1986), 111-162.

[6] A. Canonaco, The Beilinson complex and canonical rings of irregular surfaces, Mem. Amer. Math. Soc. 183 (No. 862) (2006).

[7] L. Costa And R. M. Miró-Roig, m-blocks collections and Castelnuovo-Mumford regularity in multiprojective spaces, Nagoya Math. J. 186 (2007), 119-155.

[8] L. Costa And R. M. Miró-Roig, Monads and regularity of vector bundles on projective varieties, Michigan Math. J. 55 (2007), 417-436.

[9] B. Fantechi, E. Mann and F. Nironi, Smooth toric DeligneMumford stacks, J. Reine Angew. Math. 648 (2010), 201-244. 
[10] G. Horrocks, Vector bundles on the punctured spectrum of a local ring, Proc. London Math. Soc. (3) 14 (1964), 689-713.

[11] R. Lazarsfeld, Positivity in algebraic geometry I, Ergebnisse der Mathematik und ihrer Grenzgebiete, 3. Folge, 48. Springer-Verlag, Berlin, 2004.

[12] D. Maclagan and G. Smith, Multigraded Castelnuovo-Mumford regularity, J. Reine Angew. Math. 571 (2004), 179-212.

[13] D. Mumford, Lectures on curves on an algebraic surface, Annals of Mathematics Studies 59. Princeton University Press, Princeton, N.J., 1966.

[14] G. Pareschi and M. Popa, Regularity on abelian varieties I, J. Amer. Math. Soc. 16 (2003), 285-302.

[15] J. Sidman and A. VAn TuYL, Multigraded regularity: syzygies and fat points, Beiträge Algebra Geom. 47 (2006), 67-87. 